\title{
Visual Fatigue and Performances for the 40-min Mixed Visual Work with a Projected Screen
}

\author{
Hsin-Chieh $\mathrm{Wu}^{*}$
}

Department of Industrial Engineering and Management, Chaoyang University of Technology, Taichung, Taiwan

\begin{abstract}
Objective: The main purpose of this study is to evaluate the visual angle and ambient illumination effects on visual fatigue and performances while viewing from a projected screen for 40 minutes.

Methods: Visual angle (16, 24 and 48 min arc of Chinese character height) and ambient illumination (200 and 750 lux) were the independent variables in this study. Change in critical flicker frequency (c_CFF), subjective visual fatigue, proofreading accuracy, and film-watching performance in the experimental trials were obtained from 30 young adults. Each experimental trial took 40 minutes, including a 10-min proofreading test and a 30-min film watching task.

Results: Visual angle had a significant effect on proofreading accuracy. An increased proofreading accuracy tendency was found with increasing visual angle. Higher ambient illumination (750 lux) had a positive effect on c_CFF. A significantly higher film-watching performance was also found at the higher ambient illumination (750 lux) compared to that of the lower one (200 lux).

Conclusion: We suggest that (a) the ambient illumination should be controlled at about 750 lux for better audiences' attention and visual comfort; (b) a greater visual angle (24 $48 \mathrm{~min}$ arc of character height) is more suitable for Chinese text-reading on a projected screen as compared with 16 min arc; and (c) if the visual tasks with a projected screen mostly contain video and voice, the visual angle of character height will have little effect on performance.
\end{abstract}

Keywords: Vision and lighting, information displays, office ergonomics, character size.

\section{INTRODUCTION}

The rapid development of digital science and technology has brought the digital projector to the forefront of teaching aids. Digital projector use increased immensely in both schools and companies. More people rely on digital projectors to brief plans, teach students, present papers, demonstrate products, see films or hold meetings and conferences. Nowadays, a smart classroom usually includes a computer at the teaching station, the availability of a television or video-cassette recorder, and the ability to project or send a computer signal to a large monitor, projector, or each student station. Most university schools in Taiwan are using digital projectors as an auxiliary means in teaching [1]. A large projection screen can be watched simultaneously by a large audience from different seats in a room. The viewing distance from the eye to the projection screen (more than $2 \mathrm{~m}$ ) is markedly longer than those suggested for a visual display terminal (VDT). Different viewing distances produce different subtended visual angles for a constant text size displayed on the screen. The visual angle (in min of arc) can be easily calculated by character height (in $\mathrm{mm}$ ) multiplied by 3438 and then divided by the viewing distance (in $\mathrm{mm}$ ) [2]. In other words, people who sit closer to the screen will have a greater visual angle that

Address correspondence to this author at the Department of Industrial Engineering and Management, Chaoyang University of Technology, No.168, Jifong E. Rd, Wufong District, Taichung, R.O.C. 41349, Taiwan; Tel: 886-4-2332-3000, Ext. 4537; Fax: 886-4-2374-2327;

E-mail: hcwul@cyut.edu.tw makes the projected text appear bigger. Conversely, people who sit further from the screen will experience a smaller visual angle that makes the projected text appear smaller, decreasing the text legibility.

The quantitative relationships between visual angle (text size) and Chinese text legibility have been well studied by Cai et al. [3] and Chi et al. [4]. Cai et al. [3] investigated the three commonly used Chinese font styles and indicated that the characters in the Ming style are significantly more legible than the Kai style, and the Kai style is significantly more legible than the $\mathrm{Li}$ style. Further, the number of strokes, character height, and character width can be used to predict the legibility in Chinese characters [4]. For the legibility of different fonts, Chi et al. [4] found that the $95 \%$ cumulative probability of legibility in the Hei, Ming, Kai, and $\mathrm{Li}$ styles were 20.5, 22.7, 24.6 and $25.4 \mathrm{~min}$ arc, respectively. These suggestions can be considered the minimum acceptable font size for clearly presenting Chinese characters on a VDT. It has been proven that greater visual angle of the Chinese text height will have better reading comprehension than the smaller one when reading from a computer screen [5]. Consistently throughout the literature are findings that decreasing the text legibility of a computer screen has worse readability [6].

The theoretical grounding for reading from computer screens is that the smaller visual angle (less than minimum acceptable font size) will lead to worse reading performance and more visual fatigue. The prior study [7] has also found a smaller visual angle caused a significantly worse 
proofreading performance while reading from a projection screen. Therefore, the relationships between visual angle (text size) and visual performances found in computer screens seem to be applicable to relatively larger projection screens.

The issue of visual fatigue at a computer workstation has been noted in the previous studies [8-11]. Some VDT tasks can cause symptoms of fatigue and some can result in temporary reduction of visual ability. In the near vision condition, shorter viewing distance (about $30 \sim 50 \mathrm{~cm}$ ) imposes higher eyestrain on the VDT users because of much contraction of ciliary muscle and external eye muscle for convergence and accommodation. This is not the case for the condition of far vision (with a large display), where accommodation and vergence are more or less at rest. Recently, the visual fatigue issue for viewing large-size televisions has been noted. Sakamoto, et al.'s study [15] showed that visual fatigue reached a minimum at a viewing distance of 3 times the height of the 42-inch Plasma TV. Unfortunately, most of those visual fatigue studies were focused on computer or TV screens. Studies on visual fatigue for watching from a projection screen are rare. This paper consequently deals with the visual fatigue issue for viewing a projection screen because that has not been given much recent airing so may start a debate on this potentially interesting topic. This is the main topic of the present experiment.

In addition to visual angle, the ambient illumination may be an important factor that can affect visual fatigue when watching from a projection screen. The Human Factor Society [12] recommended 200 to $500 \mathrm{~lx}$ is suitable for VDT tasks. For the cathode ray tube (CRT) display, Xu and Zhu [13] studied the effect of ambient illumination and found that performance decreased as the ambient illumination increased. Conversely, Shieh and Lin [14] indicated that the subjects had significantly better visual identification performance under the $450 \mathrm{~lx}$ condition than at the $200 \mathrm{~lx}$ level, particularly for the liquid crystal display (LCD). This is because of the differences in optical characteristics between CRTs and LCDs. However, the question remains: Does higher ambient illumination lead to less 'visual fatigue' than the lower one after watching from a projection screen? The current study also investigated the effect of ambient illumination on 'visual fatigue' for digital projector users.

The intent of this study was to investigate the effects of visual angle and ambient illumination on visual fatigue and performances for the digital projector users. Generally, the visual angle effect was investigated at a constant viewing distance, while varying the character height in $\mathrm{mm}$. However, in the context of a classroom and office meeting there is only one display and some people sit closer to the screen than others. This study intentionally maintained a constant character height and varied the viewing distance to manipulate the visual angle. Four hypotheses are proposed: (1) Visual fatigue will be increased with a greater visual angle, (2) Visual fatigue will be reduced with a higher ambient illumination, (3) Visual performance will be improved with a greater visual angle, and (4) Visual performance will be improved with a higher level of ambient illumination.

\section{METHODS}

\subsection{Subjects}

The 30 participants ( 15 men, 15 women) had a mean age of 22.4 years (range $=20$ to 24 years) and normal or corrected-to-normal vision with a visual acuity of 1.0 or better (decimal units). The subjects' visual acuity was measured using the Tumbling E chart [15] by the trained experimenter. The experimental process was explained in detail to all participants prior to participation in the study. They were then requested to fill out a written consent form to indicate their awareness of the experimental goals and their willingness to participate in this study. Each participant was paid $\$ 4.00$ (U.S.) per hour for performing the experiment.

\subsection{Apparatus}

The experimental stimuli were produced using a notebook computer (IBM X31), and presented using a digital projector (TOSHIBA TDP-T91) with a brightness of 2200 ANSI lumens and a resolution of $1024 \times 768$ pixels. The visual stimuli were projected onto a projection screen $(178 \times$ $137 \mathrm{~cm}$ ). Six lamp stands were used to control the illumination conditions in the laboratory. Each lamp had an electricity-saving light bulb with a color temperature of about $6500 \mathrm{~K}$. A palm-top illuminometer (YichangLM81LX) was used to measure the ambient illumination level on the worktable.

A handy flicker (HF, Japan) was used to measure the Critical Flicker Frequency (CFF) for the subjects. The CFF, also called flicker fusion threshold, is the transition point of an intermittent light source where the flickering light ceases and appears as a continuous light. Iwasaki and Akiya [16] confirmed that the decrease in CFF reflects a decline in retina or optic nerve activity. Because of high sensitivity and convenience, a change in CFF is considered as an important index with which to measure physiological visual fatigue $[17,18]$.

\subsection{Experimental Design}

The visual angle and ambient illumination were the independent variables. The visual angle was defined as the subtended angle of Chinese character height on the screen at a participant's eye level. We used visual angles of $16 \mathrm{~min}$ arc (which is the minimum size suggested for English capital letters), $24 \mathrm{~min}$ arc (which is the legibility threshold for the Kai-style Chinese characters), and $48 \mathrm{~min}$ arc (which is double the $24 \mathrm{~min}$ arc). The $48 \mathrm{~min}$ arc visual angle was used to examine the larger visual angle effect on proofreading accuracy. Because the Chinese character height was fixed at $41.9 \mathrm{~mm}$ here, the viewing distances of 9,6 and $3 \mathrm{~m}$ could just correspond to 16,24 , and $48 \mathrm{~min}$ arc visual angles, respectively. Two seats were arranged for each visual angle level. Fig. (1) shows the workplace arrangement in the laboratory. The subjects were not allowed to adjust their seating positions to maintain constant visual angle during the experiment.

Two levels of ambient illumination were employed: 200 lux (which is the lowest lighting level recommended for traditional VDT tasks) and 750 lux (which is a higher lighting level). The ambient illumination here was defined as 


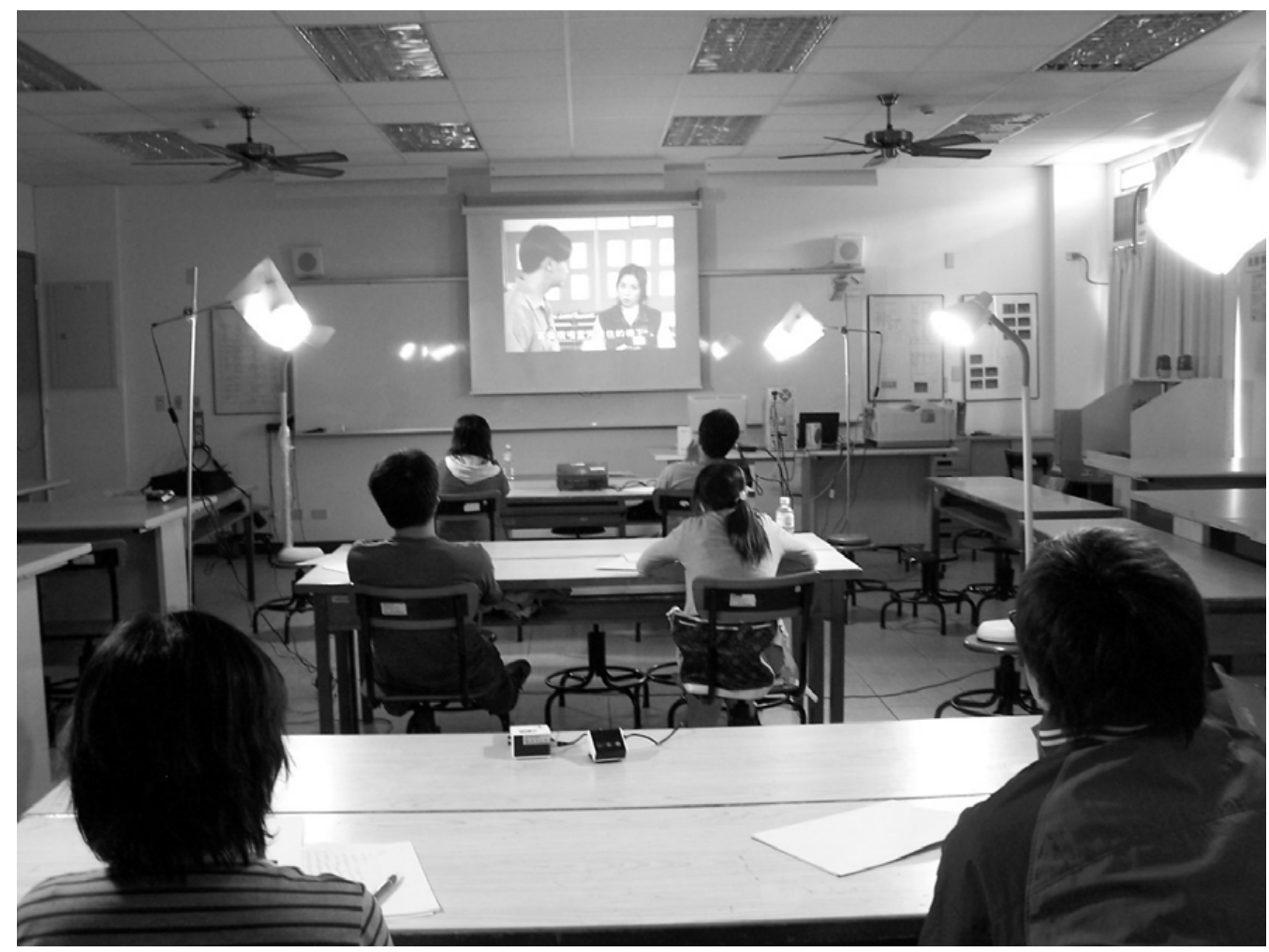

Fig. (1). The workplace arrangement used in the experiment. This picture was taken using the 750 lux ambient illumination condition.

the illumination level on the working table for the subjects. Six lamp stands, placed beside the work tables, were carefully adjusted to control the table illumination according to the experimental conditions. Lampshades were used to prevent the ambient lighting from producing glare on the projected content, as shown in Fig. (2).
Six treatments $(3 \times 2)$ comprised the visual angle and ambient illumination combinations. Thirty participants were randomly divided into three groups with each group having 10 persons, gender balanced. Each group was randomly assigned to each of the three visual angle levels (betweensubjects factor). Every subject randomly performed the two

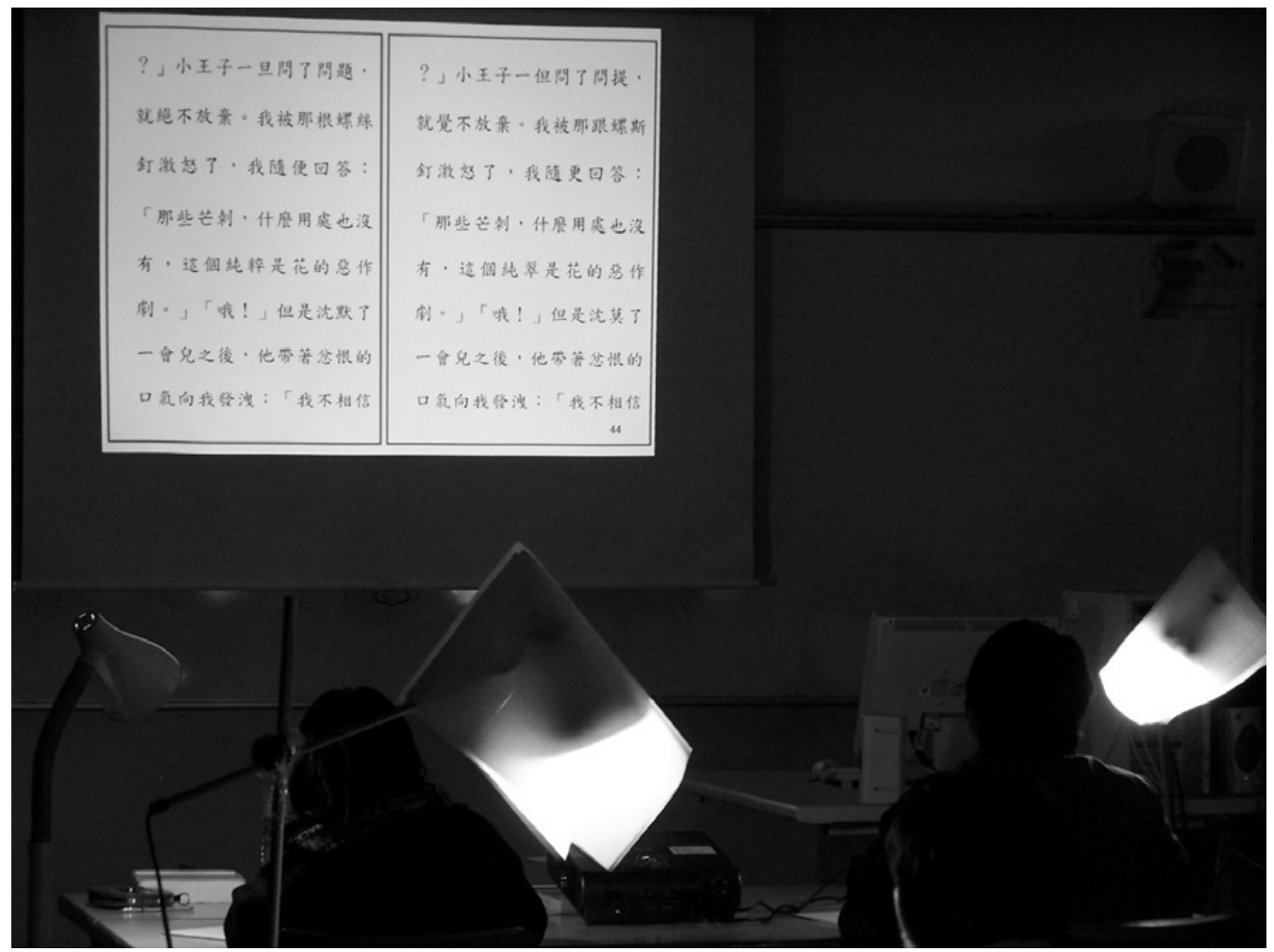

Fig. (2). The 200 lux ambient illumination condition in the experiment. 
(200 and 750 lux) ambient illumination treatments (withinsubject factor) on two different days.

\subsection{Experimental Tasks and Setup}

Each experimental trial consisted of a 10-min proofreading test and a 30-min film watching task because these two tasks are the most common work for the use of a projection screen. This study simply adopted the fixed order of these two tasks (first proofreading, then video) to simulate a real application of a digital projector. Owing to the purpose of this study was not aimed at distinguish visual fatigue between proofreading and video, the visual fatigue data were collected only after 40-min of the mixed visual works. Besides, the reason for choosing 10 minutes for the proofreading test is that a short presentation with a digital projector generally takes about $10 \sim 15$ minutes.

\subsubsection{Proofreading Test}

Twenty pages of Chinese text were sequentially presented on the projection screen one by one for $30 \mathrm{sec}$ per page in the proofreading test. Each page was designed to have left and right regions, as shown in Fig. (3). These two regions presented approximately the same text, but the right region had eight errata within the text. The proofreading errors were purely orthographic. For example: ‘待' is correct, '寺' is wrong; '頓' is correct, '噸' is wrong. The characters per region were arranged in eight lines, with about 12 characters per line. The subjects were asked to identify the errata presented on the projection screen as much as they could. They should write down the found errata on a given sheet. To maintain work motivation, subjects were paid an extra $\$ 2.00$ for individual performance above the average level for all participants. The provision of monetary incentives could let the participants continuously watch the projected text without breaks.
Several understandable Chinese articles were selected as materials for the proofreading tests. These articles were randomly assigned to different experimental trials. The characters were displayed with the 'Kai font style' in 24 point. The justification for this font selection is that Kai font style is one of the three most commonly used Chinese fonts [3]. The actual height of the characters projected on the screen was $41.9 \mathrm{~mm}$. The inter-character spacing was 12.82 $\mathrm{mm}$ and interline spacing was set at $65.98 \mathrm{~mm}$.

\subsubsection{Film-Watching Task}

Immediately following the 10-min proofreading test, participants were required to continuously watch a film for 28 minutes. The actual height of the subtitles projected on the screen was approximately the same as that of the proofreading text. At the end of the film, an attention test about the scenario in the film was completed by the subjects within 2 minutes. Eight questions were presented simultaneously on the projection screen. These questions were designed to examine whether the participants could wholly follow the film scenario. For example, 'Who dropped from the top of the hotel? (1) GARY, (2) TONY, (3) GIGI' and 'How much money did LANCHIN originally want to get? (1) 100,000 , (2) 200,000 , (3) 300,000 '. Further, the eight comprehension questions can not be answered using only the subtitles. The participants should pay attention to the images shown on the screen and listen to the audio at the same time. Therefore, the video is not extraneous. If the subject did not miss the story in the film, he or she could easily answer the questions.

In order to maintain work motivation, participants were also paid an extra $\$ 2.00$ for individual performance above average level for all participants. A "who-dun-it serial" was selected as the material for the film watching task. These materials were divided into several short films, 28-min long

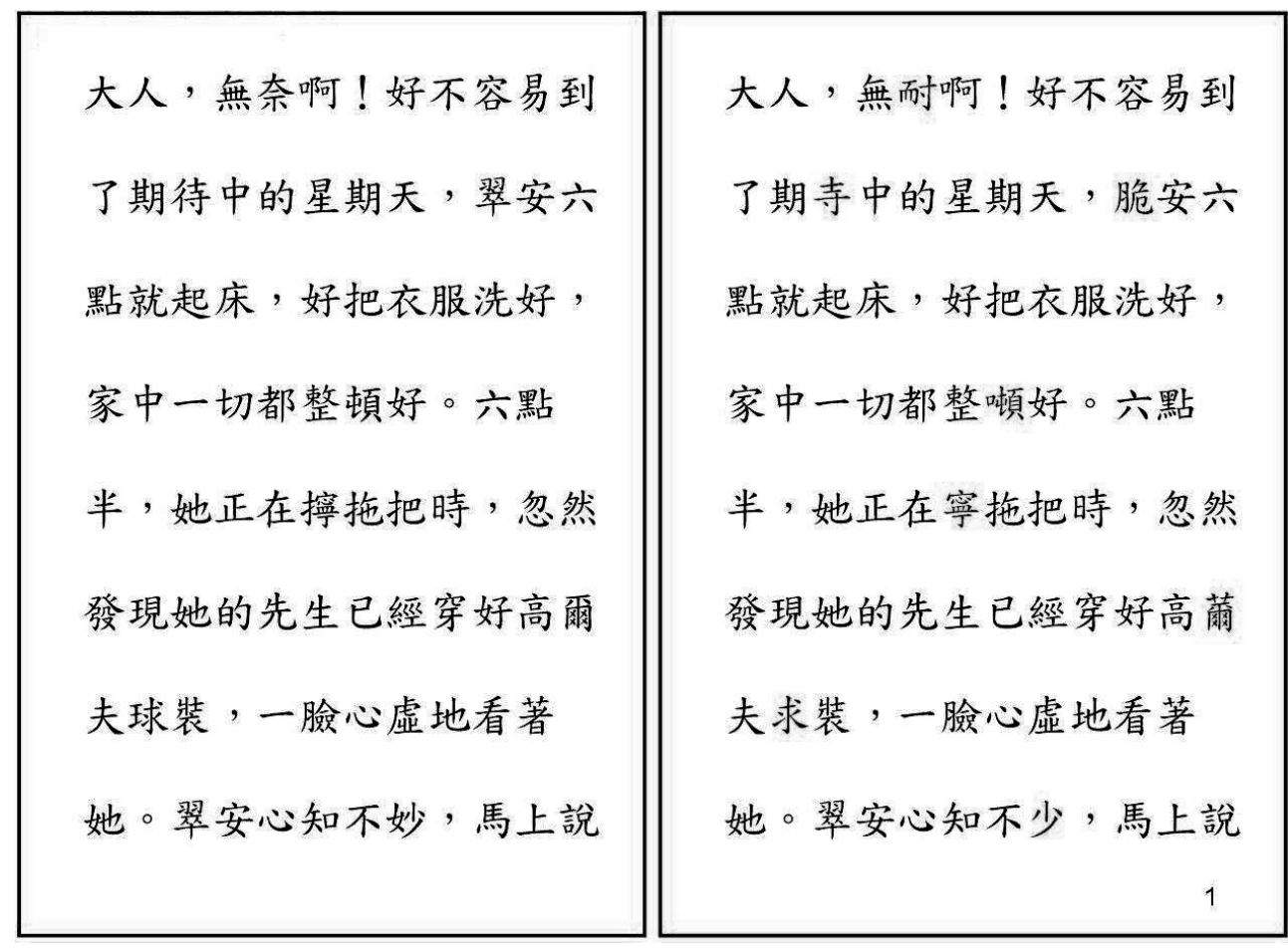

Fig. (3). The layout of the slides projected on the screen for the proofreading task. 
each. These short films were randomly assigned to different experimental trials.

\subsubsection{Experimental Setup and Controlled Factors}

The digital projector was positioned on a table $76 \mathrm{~cm}$ in height. The horizontal distance from the projection screen to the lens of the projector was $240 \mathrm{~cm}$. The elevation angle of the projector base was 11 degrees with respect to the horizontal axis. The actual size of the outline border projected onto the screen was $950 \mathrm{~mm}$ high and $630 \mathrm{~mm}$ wide. The whole projection scope was $1020 \mathrm{~mm}$ high and $1350 \mathrm{~mm}$ wide. The work tables were $76 \mathrm{~cm}$ high, $50 \mathrm{~cm}$ wide and $199 \mathrm{~cm}$ long. The illumination on the projection screen was controlled at 50 lux when the digital projector was not turned on. The text was black and the background was white for the proofreading task and their luminance ratio on the projected screen was 1:3. The light output of the digital projector was measured using the ANSI Lumen standard [19]. The projected screen was maintained at $1880 \pm 10$ ANSI Lumen. All of these set-up parameters were fixed in all of the experimental trials.

\subsection{Dependent Measures}

Each experimental trial collected four dependent variables, the change in $\mathrm{CFF}$, rating in visual fatigue, proofreading accuracy, and film-watching performance. These variables are mentioned below:

The change in CFF (c_CFF) was analyzed to determine the visual fatigue for the participants. Eye fatigue here was defined by a decrease of at least $1 \mathrm{~Hz}$ of CFF value for the participants. The CFF measurement taken before the trial was subtracted from that taken after the 40-min of the mixed visual work. Two values were collected for each CFF measurement. The first was obtained when the flicker frequency changed upward (from 1 to $79 \mathrm{~Hz}$ ) while the second was gathered when the frequency changed downward (from 79 to $1 \mathrm{~Hz}$ ). These two values were averaged as the result for one measurement.

The rating in visual fatigue was the perceived eyestrain determined by the participants after the 40-min of the mixed visual work. A 10-point scale, with 1 representing "not at all" and 10 representing "extremely visual fatigue" was used.

Proofreading accuracy was the ratio of the number of correctly found errata to the total given errata in the 10-min proofreading test. Because each page was presented on the projection screen for only 30 seconds, the subjects had a little time pressure to proofread the entire text during the 10 minutes. In this way, the completion time for the proofreading test was not collected.

The film-watching performance was defined as the ratio of the number of correctly answered questions to the total number of questions in the attention test.

\subsection{Experimental Procedure}

\subsubsection{Screening Test}

To evaluate the differences among the three subject groups (named A, B, and C), all of the subjects performed a 40 -min screening test at the same visual angle (24 min arc) and under the same ambient illumination condition (450 lux). The screening test was designed to make sure that the subject group effect would not affect the total results. After the participants had assumed a comfortable sitting posture, the subject's CFF values were measured. They then began to perform the proofreading test. Participants were asked to identify the errata in the text as much as they could and write down the errata found on a sheet provided. After 10-min proofreading, the 30-min film-watching task began. The participants' CFF values were then measured again after the film-watching task. After the test, rating in visual fatigue of the subject was also collected.

\subsubsection{Experimental Phase}

Every subject performed two 40-min experimental trials on two separate days under two different ambient illumination conditions (200 and $750 \mathrm{lux}$ ). The trial order was randomized. Groups A, B, and $\mathrm{C}$ were assigned to visual angles of 16, 24, and $36 \mathrm{~min}$ arc, respectively. Participants were seated with the assigned visual angle during the experimental trials. The work procedure for each experimental trial was the same as that used in the screening test.

\subsection{Data Analysis}

SPSS 10.0 Statistical Software was used for the collected data analyses. Before conducting the analysis of variance (ANOVA), the normality assumptions for the data distribution were checked using Kolmogorov-Smirnov Goodness-of-Fit Test. The significance level was set at $p<$ 0.05. If the data distribution was normal, a mixed-factor ANOVA was used to determine the visual angle (three levels, between-subjects) and ambient illumination (two levels, within-subject) effects on the dependent measures, with the LSD method for post-hoc pairwise comparisons. Effects were considered 'significant' when $p<0.05$. If the respondent data distribution was not normality, a proper nonparametric test should be used to evaluate the studied main effects.

\section{RESULTS}

Table 1 shows the descriptive statistics (mean \pm standard deviation) for each dependent variable for the three subject groups in the screening test. The one-way ANOVA test results showed that the subject group had very little effect on all of the dependent variables. These results merely confirmed that the group effect would not confound the between-subject factor (visual angle) in the following experiment.

For the experiment results, mean and standard deviation values for the c_CFF, rating in visual fatigue, proofreading accuracy, and film-watching performance under each independent variable level are shown in Table 2. There was a tendency for increased eye fatigue, as measured by c CFF, with increasing visual angle. Further, more decrease in CFF was found at the $200 \mathrm{lux}(-2.28 \mathrm{~Hz})$, as compared with that at the 750 lux $(-1.17 \mathrm{~Hz})$. The mean ratings in visual fatigue were similar among all the experimental treatments. The proofreading accuracy was $57.1 \%, 63.5 \%$, and $75.7 \%$ for the visual angles 16, 24, and $48 \mathrm{~min}$ arc, respectively. There was a tendency toward increased proofreading accuracy with increasing visual angle. The responded proofreading accuracy between the 200 and 750 lux conditions were similar (about 65\%). It is interesting that the respondent 
Table 1. Screening Test Results

\begin{tabular}{|lccccc|}
\hline \multicolumn{1}{|c}{ Group } & $\mathbf{n}$ & c_CFF (Hz) & Rating in Visual Fatigue & Proofreading Accuracy (\%) & Film-Watching Performance (\%) \\
\hline \hline Group A & 10 & $-1.33 \pm 1.39$ & $3.3 \pm 1.6$ & $66.3 \pm 11.7$ & $73.0 \pm 14.4$ \\
Group B & 10 & $-1.62 \pm 1.58$ & $3.5 \pm 1.8$ & $64.9 \pm 13.3$ & $74.4 \pm 14.3$ \\
Group C & 10 & $-1.53 \pm 1.46$ & $3.0 \pm 1.2$ & $65.8 \pm 10.9$ & $72.1 \pm 12.3$ \\
\hline ANOVA* & & & & 0.036 \\
$F(2,27)$ & & 0.229 & 0.257 & 0.965 & 0.064 \\
$p$ & & 0.797 & 0.775 & 0.939 \\
\hline
\end{tabular}

*The one-way ANOVA was conducted to test the group effect on each dependent variable. $p<0.05$ The significance level was set at $p<0.05$.

film-watching performance at 750 lux illumination level $(81.7 \%)$ was greater than that at 200 lux illumination level $(72.5 \%)$.

All p-values obtained from the Kolmogorov-Smirnov $\mathrm{Z}$ test exceeded 0.05 , supporting the normality assumptions for the following analyses of variances (ANOVAs). Based on the ANOVA results summary (Table 3 ), visual angle had a significant effect on proofreading accuracy $(\mathrm{p}<0.05)$. Ambient illumination had a significant effect on both c_CFF and film-watching performance $(p<0.05)$. The interaction effect was not significant on all of the dependent variables, suggesting that the visual angle and ambient illumination effects were independent.

Fig. (4) illustrates the post-hoc test (LSD method) results for the difference in proofreading accuracy among the three visual angle levels. There existed a significant difference in proofreading accuracy between the $48 \mathrm{~min}$ arc and $24 \mathrm{~min}$ arc conditions $(p=0.009)$. A significant difference was also found between the $48 \mathrm{~min}$ arc and $16 \mathrm{~min}$ arc conditions $(\mathrm{p}<0.01)$. However, no significant difference was obtained between the 16 and $24 \mathrm{~min}$ arc conditions.

\section{DISCUSSION}

Our participants were able to assume a normal sitting posture with 90 degrees of the knee joint because the used chairs were adjustable for the people's specific anthropometrics. The projection area has a dark illumination level (50 lux), and the projector produced clear text and images on a bright background screen. The visual angles and the ambient illuminations were within the usual range for that in classrooms or meeting rooms. Based on these conditions, our experimental tasks resembled typical viewing conditions for work or study with a projection screen. It would be feasible to apply the experimental results in a practical workplace. The section below discusses the results arising directly from the experiment. Some important findings and recommendations for the use of a projection screen are described.

Table 2. Dependent Variable Results Under Each Independent Variable Level

\begin{tabular}{|lccccc|}
\hline Independent Variable & $\mathbf{n}$ & $\mathbf{c} \_$CFF (Hz) & Rating in Visual Fatigue & Proofreading Accuracy (\%) & Film-Watching Performance (\%) \\
\hline \hline Visual angle & & & & & \\
16 min of arc & 20 & $-1.37 \pm 1.40$ & $3.4 \pm 1.7$ & $67.1 \pm 16.4$ & $71.9 \pm 17.1$ \\
24 min of arc & 20 & $-1.60 \pm 1.85$ & $3.1 \pm 1.2$ & $63.5 \pm 14.6$ & $79.4 \pm 15.3$ \\
48 min of arc & 20 & $-2.20 \pm 1.77$ & $3.5 \pm 1.9$ & $66.7 \pm 10.6$ & $80.0 \pm 12.4$ \\
\hline Ambient illumination & & & & $64.9 \pm 15.3$ & $81.7 \pm 12.6$ \\
200 lux & 30 & $-2.28 \pm 1.73$ & $3.2 \pm 1.6$ & 6.5 \\
750 lux & 30 & $-1.17 \pm 1.48$ & $3.3 \pm 1.5$ & & \\
\hline
\end{tabular}

Table 3. Summary of the ANOVA Results

\begin{tabular}{|lcccc|}
\hline Variance Source & c_CFF (Hz) & Rating in Visual Fatigue & Proofreading Accuracy (\%) & Film-Watching Performance (\%) \\
\hline \hline Between subjects & & & $*$ & NS \\
Visual angle (V) & NS & NS & & $*$ \\
Within subject & & NS & NS \\
Ambient illumination (A) & $*$ & NS & NS \\
V $\times$ A & NS & & \\
\hline p $<0.05$ significant level.
\end{tabular}

\footnotetext{
$* \mathrm{p}<0.05$ significant level.
} 


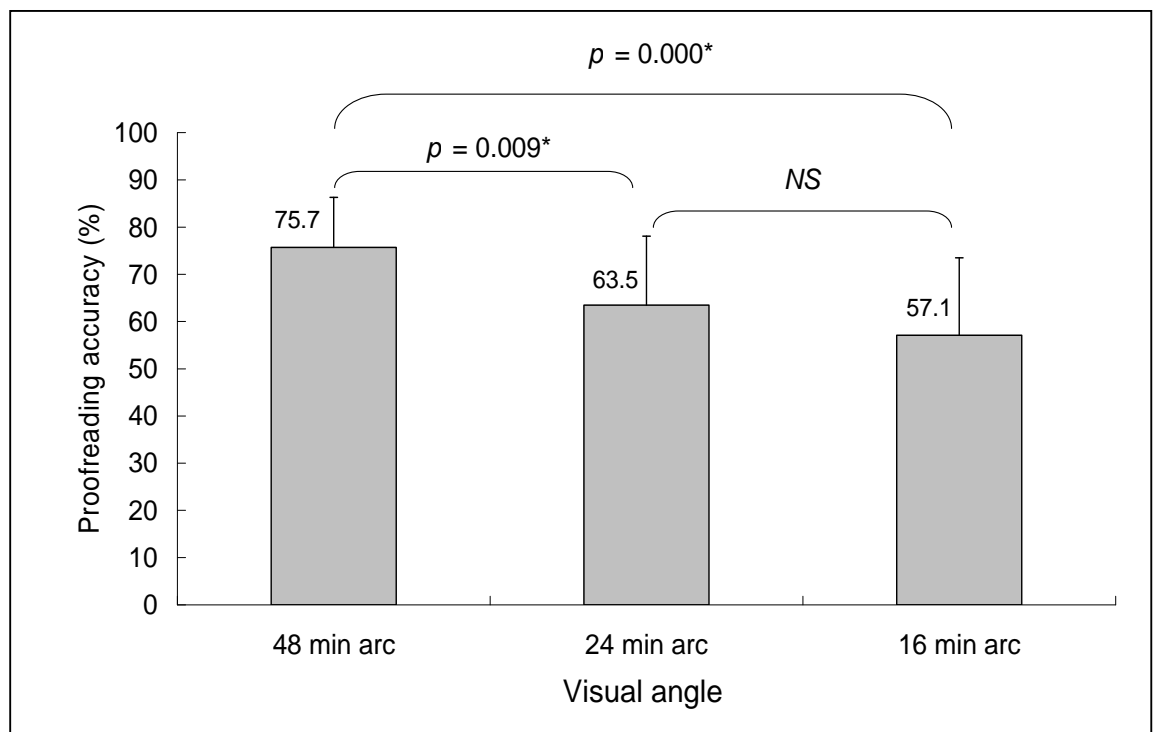

Fig. (4). Proofreading accuracy results under the three visual angles. Comparison was made with standard post-hoc tests (LSD method). * is significant; NS is not significant at $\alpha=0.05$.

\subsection{CFF Measure Versus Subjective Visual Fatigue}

This study intentionally maintained a constant character height and varied the viewing distance (i.e. 3, 6, and $9 \mathrm{~m}$ ) to manipulate three visual angle levels. Based on the measurement results, the c_CFF (about -1.37 to $-2.2 \mathrm{~Hz}$ ) did not significantly differ among the three levels of visual angle. This result is not consistent with $\mathrm{Wu}$ et al.'s [20] finding that a smaller angular character size (visual angle) leads to more decrease in CFF after 100-min of reading work. This inconsistency could be explained by the fact that the experimental work of the present study contained 10-min proofreading and 30-min video watching, but that of $\mathrm{Wu}$ et al.'s study [20] was 100-min reading. Further, both the viewing distance and the display type were also different between the present study and Wu et al.'s study [20].

Because eye fatigue here was defined by a decrease of at least $1 \mathrm{~Hz}$ of $\mathrm{CFF}$ value, all of the treatment conditions caused more than $1 \mathrm{~Hz}$ CFF decrease implying that visual fatigue occurred after the 40-min mixed visual work. Further, a significant decrease in CFF was found under the lower illumination level (200 lux). Otherwise, the collected ratings of visual fatigue under all of the treatment conditions are similar (about 3 in the 10-point scale). That means the significant decrease in CFF was not reflected in subjective visual fatigue. This may be due to CFF measurement is more sensitive than rating of visual fatigue. Decrease in CFF reflects a decline in retina or optic nerve activity, but not necessarily caused by visual workload, mental and muscular workload can induce the same result [16]. Because of the small ratings in visual fatigue found in this study, the significant decrease in CFF could be partly caused by the mental workload of the proof-reading task and film watching task.

The reasons why the collected subjective visual fatigue were little may be: (1). Working time in each trial was only 40 minutes that will not cause great subjective visual fatigue; (2). The viewing distances were set at 3 to 9 meters, which are much longer than $50 \mathrm{~cm}$ in general for VDT tasks.
Longer distance might cause less eyestrain in visual task; (3). The film watching task was taken after proof-reading task that may have a relaxing effect in the visual adjustment process. Film watching task needs less fixation effort at text than proof-reading task that may relieve visual tension caused by proof-reading task. But these contentions are required to be proven in the further research.

\subsection{Visual Angle Effect on Proofreading Performance}

When proof-reading Chinese text from a projection screen, the results of this study suggested that visual angle of 48 min arc would lead to significantly better accuracy than those at 16 and $24 \mathrm{~min}$ arcs. This study confirmed that the visual angle effect was significant in proofreading performance when using a projection screen. The current results strongly suggest that a larger visual angle is proposed if the text legibility is the most important for watching a projected context. In other words, increasing the angular letter size (also means sitting close to the projection screen) can improve reading-related performance. This finding is consistent with those of the VDT-based studies before [1-3]. The results derived from this study can be applied in the design of Chinese text size for conducting a short presentation with a digital projector.

For the illumination effect, little difference in proofreading accuracy was found between the 200 lux and the 750 lux conditions. This is because the ambient lighting was well controlled in this study to maintain a constant luminance contrast ratio $\left(\mathrm{L}_{\text {background }} / \mathrm{L}_{\text {text }}\right.$ ) for the projected text throughout the experiment. Therefore, the legibility of the projected text was similar between 200 and 750 lux conditions. The participants performed the 10-min proofreading test at the beginning of the trial and thus the performance would hardly be affected by the later eye fatigue derived from the lower ambient illumination. Further research is required to confirm this suggestion in the case of prolonged proofreading from a projection screen. 


\subsection{Ambient Illumination Effect on Film-Watching Performance}

This study found that the higher ambient illumination (750 lux) resulted in a significantly better film-watching performance than the lower ambient illumination (200 lux). This finding was consistent with Shieh and Lin's study [10], which suggested that maintaining a brighter ambient illumination level (about $450 \mathrm{lux}$ ), rather than reducing it to a lower level (200 lux). Based on our experimental settings, the luminance contrast ratio $\left(\mathrm{L}_{\text {background }} / \mathrm{L}_{\text {text }}\right)$ of the projected text was controlled at $3: 1$. It seems that the ambient illumination would be very unlikely to have any effect on screen luminance contrast ratio. Therefore, the decrease in film-watching performance is due mostly to the darker ambient illumination in the room. The 200 lux condition produced a higher contrast between bright screen and dark room (as shown in Fig. 2). The ambient lighting will affect the reflection of light from the wall behind the screen. Whilst the focus of the subject would be on the screen their obvious wider field of view would mean that any contrast around the screen (i.e. between the screen and the rear wall) would affect eye fatigue. It is therefore no surprise that a higher ambient illumination (hence a reduced contrast between the screen and light reflected from the rear wall), lead to reduce fatigue. Consequently, the respondent attention performance and eye fatigue level were worse under the 200 lux condition than at 750 lux. This result strongly implied that adequate ambient illumination would bring better performance and less eye fatigue. We therefore suggest that it is not advantageous to maintain darkness throughout the room when watching videos with a projection screen.

The film-watching performance shows a trivial difference among the three visual angles. This can be explained by the task characteristics of watching a film. The visual stimuli of a film were mostly dynamic images instead of static text. Participants could easily receive the story of the film by mainly watching the whole video, instead of only reading the subtitles. These are the reasons why subjects had similar film-watching performance under different visual angles. Therefore, the visual angle effect was not significant on performance for watching a film with a projection screen.

\subsection{Limitations and Future Research}

The proofreading tests were performed using a big projection screen. This is different from VDTs and computer screens. This study found that increasing the angular character size (by sitting close to the projection screen) could improve proofreading performance. This finding is consistent with those of the previous VDT-based studies. The experimental results indicated that visual angle of 48 min arc led to significantly better accuracy than those of 16 and 24 min of arcs. Because only three different visual angles $(16,24$ and $48 \mathrm{~min}$ arc) were tested in this study, we cannot make sure $48 \mathrm{~min}$ arc is the optimal value for all angular character sizes. For a VDT with a luminance contrast of 50:1, Chi [7] suggested that the Kai-style Chinese character size should be 24.6 min arc for $95 \%$ legibility. For an electronic paper display, a larger visual angle of $33 \mathrm{~min}$ arc has been recommended because of its obvious lower luminance contrast of 1.2:1 [21]. Different display types have different resolutions and luminance contrast ratios. The legibility threshold for Chinese characters may be different among various display types. Consequently, further research is required to investigate the optimal visual angle for reading from a projection screen because projection screen characteristics are very different from that of a VDT or an ebook display.

This study manipulated visual angle by having participants sit at different distances from the screen because the projected image size was held constant. Someone may argue that the visual angle manipulation here is perfectly confounded with viewing distance. In order to untangle the visual angle or distance effects, Lo [22] evaluated visual fatigue and performance under different distances using the same visual angle. His research results indicated that the distance effect was not significant on both visual fatigue and performance. Therefore, the visual angle effect in the current study is contributed mostly by the letter size effect, instead of the distance effect.

Although some findings in this study are consistent with those from the VDT-based studies, announcing that all of the suggestions for VDTs can be applied in using a projection screen is premature. Some other factors such as screen color, brightness, resolution and luminance contrast were not discussed in this study. These factors may result in different performance between a projection screen and a VDT. When watching a projection screen, the head elevation angle is usually upward, which is markedly different from using a VDT (head upright or downward). Therefore, further research is required to confirm whether previous guidelines for using a VDT can be wholly applied in the use of a projection screen.

\section{CONCLUSION}

This paper examined the ergonomic issues in using a projection screen that have not been given much recent attention. The significant effect of visual angle found in this study was similar to those discovered from VDT-based studies. The experimental results suggested that a greater visual angle (about $48 \mathrm{~min}$ arc) had the best performance for proofreading Chinese text on a projection screen. Significantly higher film-watching performance and less visual fatigue were found at 750 lux ambient illumination compared to 200 lux. These findings can be applied when determining the viewing position or ambient illumination when using a digital projector. For examples, the audiences are recommended to sit in the front seats for better visual performance; the ambient illumination should be controlled at about 750 lux for better audiences' attention and visual comfort; to install another projection screen in the middle of a large room is required for the rear audiences. More projection related studies should be conducted to understand whether other factors (e.g., luminance contrast and color combinations of text and background) would affect visual performance and fatigue when watching a projection screen.

\section{ACKNOWLEDGMENTS}

The author acknowledges the National Science Council of the Republic of China (Contract No: NSC94-2213-E-324011) for financially supporting this research. The author also thanks $\mathrm{Yu}$-Ching Cheng for her great assistance in laboratory experiment execution. 


\section{CONFLICT OF INTEREST}

Declared none.

\section{REFERENCES}

[1] Bai YW, Liu YC, Tsai CH. Using image processing methods to reduce dazzle in the eyes from a digital projector. IEEE Instrumentation and Measurement Technology Conference; Hangzhou: China 2011; pp. 1481-6.

[2] Sanders MS, McCormick EJ. Human factors in engineering and design. $7^{\text {th }}$ ed. New York: McGraw-Hill 1993; pp. 102-15.

[3] Cai D, Chi CF, You M. The legibility threshold of Chinese characters in three-type styles. Int J Ind Ergon 2001; 27: 9-17.

[4] Chi CF, Cai D, You M. Applying image descriptors to the assessment of legibility in Chinese characters. Ergonomics 2003; 46: 825-41.

[5] Chan AHS, Lee PSK. Effect of display factors on Chinese reading times, comprehension scores and preferences. Behav Inf Technol 2005; 24: 81-91.

[6] Bernard ML, Chaparro BS, Mills MM, Halcomb CG. Comparing the effects of text size and format on the readability of computerdisplayed Time New Roman and Arial text. Int J Hum Comput Stud 2003; 59: 823-35.

[7] Wu HC, Cheng YC, Uang ST. Effects of viewing distance and local illumination on projection screen visual performance. Int $\mathrm{J}$ Appl Sci Eng 2011; 9: 1-11.

[8] IBM. IBM human factors of workstations with visual display. $3^{\text {rd }}$ ed. San Jose, CA: International Business Machines Corporation 1984.

[9] Tyrrell RA, Leibowitz HW. The relation of vergence effort to reports of visual fatigue following prolonged near work. Hum Factors 1990; 32: 341-57.

[10] Jaschinski-Kruza W. Eyestrain in VDU users: viewing distance and resting position of ocular muscles. Hum Factors 1991; 33: 69-83.

[11] Jaschinski W. Fixation disparity at different viewing distances and the preferred viewing distance in a laboratory near-vision task. Ophthalmic Physiol Opt 1998; 18: 30-9.
[12] American National Standard Institute (ANSI). American national standard practice for human factors engineering of visual display terminal workstations. ANSI/HFS Standard no. 100-1988. Santa Monica, CA: Human Factors and Ergonomics Society 1988.

[13] $\mathrm{Xu} \mathrm{W}, \mathrm{Zhu} \mathrm{Z}$. The effects of ambient illumination and target luminance on color coding in a CRT display. Ergonomics 1990; 33 : 933-44.

[14] Shieh KK, Lin CC. Effects of screen type, ambient illumination, and color combination on VDT visual performance and subjective performance. Int J Ind Ergon 2000; 26: 527-36.

[15] Taylor HR. Applying new design principles to the construction of an illiterate E chart. Am J Optom Physiol Opt 1978; 55: 348.

[16] Iwasaki T, Akiy S. The significance of changes in CFF values during performance on a VDT-based visual task. In: Kumashiro M, Megaw ED, Eds. Towards human work: Solutions to problems in occupational health and safety. London: Taylor \& Francis 1991; pp. 352-7.

[17] Chi CF, Lin FT. A comparison of seven visual fatigue assessment techniques in three data-acquisition VDT tasks. Hum Factors 1998; 40(4): $577-90$

[18] Iwasaki T, Kurimoto S, Noro K. The changes in colour critical flicker fusion (CFF) values and accommodation times during experimental repetitive tasks with CRT display screens. Ergonomics 1989; 32(3): 293-305.

[19] ANSI. International standard: Electronic projection-fixed resolution projectors. Geneva, Switzerland: IEC., No. IT7.228; 1997.

[20] Wu HC, Lee CL, Lin CT. Ergonomic evaluation of three popular Chinese e-book displays for prolonged reading. Int J Ind Ergon 2007; 37: 761-70.

[21] Wu $\mathrm{HC}$, Chan $\mathrm{CCH}$. E-book character size design for young adults in Taiwan. WSEAS Trans Inf Sci Appl 2006; 3: 2159-64.

[22] Lo YC. Effect of display type and viewing distance on visual performance and visual fatigue. Master Thesis. Taichung: Chaoyang University of Technology (in Chinese) 2010.

This is an open access article licensed under the terms of the Creative Commons Attribution Non-Commercial License (http: //creativecommons.org/licenses/ by-nc/3.0/) which permits unrestricted, non-commercial use, distribution and reproduction in any medium, provided the work is properly cited. 